\title{
Review Article \\ Interaction among Cells of Bone, Immune System, and Solid Tumors Leads to Bone Metastases
}

\author{
Ilaria Roato \\ CeRMS, A.O. Città della Salute e della Scienza, Via Santena 5, 10126 Torino, Italy \\ Correspondence should be addressed to Ilaria Roato; roato78@libero.it
}

Received 26 February 2013; Accepted 8 April 2013

Academic Editor: Giacomina Brunetti

Copyright (C) 2013 Ilaria Roato. This is an open access article distributed under the Creative Commons Attribution License, which permits unrestricted use, distribution, and reproduction in any medium, provided the original work is properly cited.

Bone metastases are a dismal consequence for different types of solid tumors, such as breast, prostate, lung, and kidney cancer. The mechanisms regulating the interactions among bone, immune system, and tumor cells have been deeply investigated, and many studies are ongoing to define the specific role of the different cells in the bone metastatic process. The affinity of some tumors to growth in bone results from the special microenvironment provided by bone. Moreover, immune system and bone have a bidirectional relationship: bone cells express surface molecules ruling the expansion of hemopoietic stem cells from which all cells of the mammalian immune system derive, and various immunoregulatory cytokines influence the fate of bone cells. The last findings allow to extend the concept of vicious cycle and add T cells as mediators of the tumor growth in bone.

\section{Introduction}

Bone metastases are a common cause of morbidity in patients affected by different types of cancer and are classified in osteolytic (bone resorbing), osteoblastic (bone forming), and mixed, containing both elements. The presence of the mixed lesions suggests that the processes of bone resorption and formation may occur together and are not mutually exclusive activities. Bone metastases may occur many years after the primary tumor and have become a chronic condition for many patients with advanced cancers markedly affecting their quality of life and the demands on health care resources $[1,2]$. Certain tumor types such as breast and prostate cancer show a high incidence for metastasis to bone, and a significant proportion of patients with advanced cancer of the lung, kidney, and thyroid have skeletal involvement [1]. Osteoclasts (OCs) are the main responsible for the bone destruction in osteolytic lesions, even though their activation varies depending on the tumors. OCs are multinucleated cell of hematopoietic origin residing in bone and their main activity is represented by the resorption of the mineralised bone matrix [3]. OCs attach themselves to bone, creating a microenvironment between itself and the underlying bone matrix, a specialized structure called sealing zone. This compartment is acidified by an electrogenic proton pump (H+-ATPase) and a Cl-channel in order to solubilize the mineral component of bone, exposing its organic matrix, consisting largely of type I collagen, which is subsequently degraded by cathepsin $\mathrm{K}$. To facilitate the resorption process, OCs polarize their structure and form the ruffled border, which allows the active transport of $\mathrm{H}+$ ions through the vacuolar proton pump $[4,5]$.

Osteoblastic metastases are prevalent in advanced prostate cancer patients and induced by cancer cell interactions with osteoblasts (OBs) and their progenitors, by production of transforming growth factor- $\beta$ (TGF- $\beta$ ), bone morphogenetic protein, insulin-like growth factor (IGF), fibroblast growth factor (FGF), and WNTs [6]. OBs respond to morphogenetic factors by activating SMAD signalling, to growth factors by MAPK and PKC signalling, and to WNT by $\beta$ catenin-regulated pathways. These pathways converge on and interact with the RUNX2 transcriptional network, which drives OBs differentiation and proliferation.

Here, I review the current knowledge on the interactions between immune system and solid tumors in promoting bone metastasis formation.

\section{Bone and Immune System Crosstalk}

Bone, hematopoietic, and immune systems are in deep physical contact and share several common pathways. OC precursors $\mathrm{T}$, and $\mathrm{B}$, and NK cells arise from the same stem cell, thus 
some of the receptors and ligands that mediate the immune process also rule the maturation of OC precursors and the ability of the mature cell to degrade bone. Immune system and bone have a bidirectional relationship: bone cells express surface molecules ruling the expansion of hemopoietic stem cells from which all cells of the mammalian immune system derive, and various immunoregulatory cytokines influence the fate of bone cells $[7,8]$. OC precursors circulate among the peripheral blood mononuclear cells (PBMCs), which act as a reservoir for replenishing the pre-OC pool in the bone marrow and also as a potentially abundant source of pre-OCs that can be recruited into bone or joint tissue in response to reparative or pathological signals.

A crucial molecular link between the immune system and bone is represented by the receptor activator of nuclear factor NF-kB ligand (RANKL), its receptor RANK, and the natural decoy receptor osteoprotegerin (OPG). The membrane RANKL is expressed by OBs/stromal cells, and the soluble RANKL is secreted by activated T cells $[9,10]$, whereas the receptor RANK is expressed on OC precursors and also on tumor cells [11]. The RANKL to OPG ratio in serum has been suggested to be the critical factor in determining $\mathrm{OC}$ activation at bone level, with higher serum RANKL to OPG ratio being a marker for upregulation of osteoclastogenesis [12].

The RANKL production by activated $\mathrm{T}$ cells can directly regulate osteoclastogenesis and bone remodelling, and it explains why different pathological conditions, such as cancer, result in systemic and local bone loss. A host of immune factors, including costimulatory receptors, cytokines such as interferon- $\gamma$ (IFN- $\gamma$ ) and tumor necrosis factor (TNF), and $\mathrm{T}$ and $\mathrm{B}$ lymphocytes play a fundamental role in the regulation of bone cell development and bone turnover, and in pathogenesis of bone disease [13]. IFN- $\gamma$ has a controversial role in osteoclastogenesis because it has an antiosteoclastogenic effect in vitro [14] and in vivo in animal studies [15], whereas in humans it increases in oestrogen deficiency and in rheumatoid arthritis with bone loss [16, 17]. IFN$\gamma$ influences osteoclastogenesis both directly and indirectly: it targets maturing OC, thus blocking OC formation [18] and it stimulates T-cell activation, thus proosteoclastogenic factors increase [19]. T cells also produce interleukin-7 (IL7), a cytokine with different effects on hematopoietic and immunologic systems. IL-7 support B, and T lymphopoiesis [20], and it is also important for the correct bone homeostasis $[21,22]$. Some studies demonstrated that IL-7 promotes osteoclastogenesis by upregulating T-cell-derived cytokines, such as RANKL [23-25] and that its production is increased by oestrogen deficiency [26]. Recently investigators focused on the OC modulatory activity of T cells, showing that OCs are able to present antigenic peptides to $\mathrm{T}$ cells and to induce FoxP3 expression in CD8+ T cells, which rule an inappropriate activation of the immune response [27]. The cellular responses in cell-to-cell interactions between $\mathrm{T}$ cells and OCs are regulated through reciprocal CD137/CD137L and RANK/RANKL interactions [28]. CD137 is a costimulatory member of the TNF receptor induced by T-cell receptor activation. Its ligand CD137L is expressed on OC precursors: in vitro CD137L ligation suppresses osteoclastogenesis through the inhibition of OCs precursor fusion [28]. On the other hand, RANKL expressed on T cells binds to RANK on OCs, producing a reverse signal in $\mathrm{T}$ cells able to enhance apoptosis.

\section{The Interplay among Bone and Tumor Cells}

The affinity of some tumors to growth in bone results from the special microenvironment provided by bone. These local interactions between tumor cells and bone form a vicious cycle, which underlies the development of skeletal metastases (Figure 1) [29]. Bone marrow is a favourable soil for some tumor cells, which have a biological proclivity for this tissue. For instance, bone marrow produces factors, such as CXCL12, with a chemotactic role on cancer cells, which, on the other hand, express the chemokine receptors, CXCR4 and CXCR7 [30, 31]. Moreover, activated OCs resorb bone and release growth factors enmeshed in the bone matrix, such as bone morphogenetic proteins, TGF- $\beta$, insulin-like growth factor, fibroblast growth factor, and others that stimulate the growth of metastatic tumor cells [32]. Cancer cells, in turn, secrete prostaglandins, parathyroid hormone, parathyroid hormonerelated peptide, activated vitamin D, interleukin-6 (IL-6), and TNF that may lead to an increase in RANKL expression on OBs and bone marrow stromal cells [3], which stimulates the OC number, survival, and activity, promoting osteolytic metastases. Notch-Jagged interactions in the bone marrow suggest direct activation of osteolysis by cancer cells through this unique interaction. In particular, Jagged1, which is a downstream mediator of the prometastatic TGF- $\beta$, promotes tumor growth through stimulation of IL- 6 production from OBs, and directly it activates OC differentiation [33]. Moreover, Jagged 1 is overexpressed by bone metastatic tumor cells [34], whereas its receptor Notch is frequently expressed by progenitors and mature cells in the bone marrow [35]. In breast cancer, Notch-Jagged interactions activate biological responses in OCs and OBs, which promote both tumor invasion of bone and tumor cell growth in the bone [33].

Interestingly, prostate and breast cancer cells show osteomimicry, that is, the ability to acquire a bone cell phenotype [36-38]. Breast and prostate tumor cells respond to growth factor stimulation via activation of various osteoblastic transcription factors. This would suggest that bone lesions may also occur through differentiation of the cancer cells towards an osteoblastic bone-forming phenotype, which is a phenomenon that has been observed in the bone metastatic prostate and breast cancer celllines $[39,40]$.

\section{T Cells as Regulator of Tumor Growth in Bone}

Recently, the concept of vicious cycle has been enlarged to include T cells as additional regulators of bone tumor growth, regardless of the OC status [41]. Blockade of OC activity efficiently decreases tumor burden as well as associated bone lesions in immune-compromised animals bearing human osteolytic cancers. Despite the antiresorptive therapies are efficient in blocking the OCs, part of the treated patients 


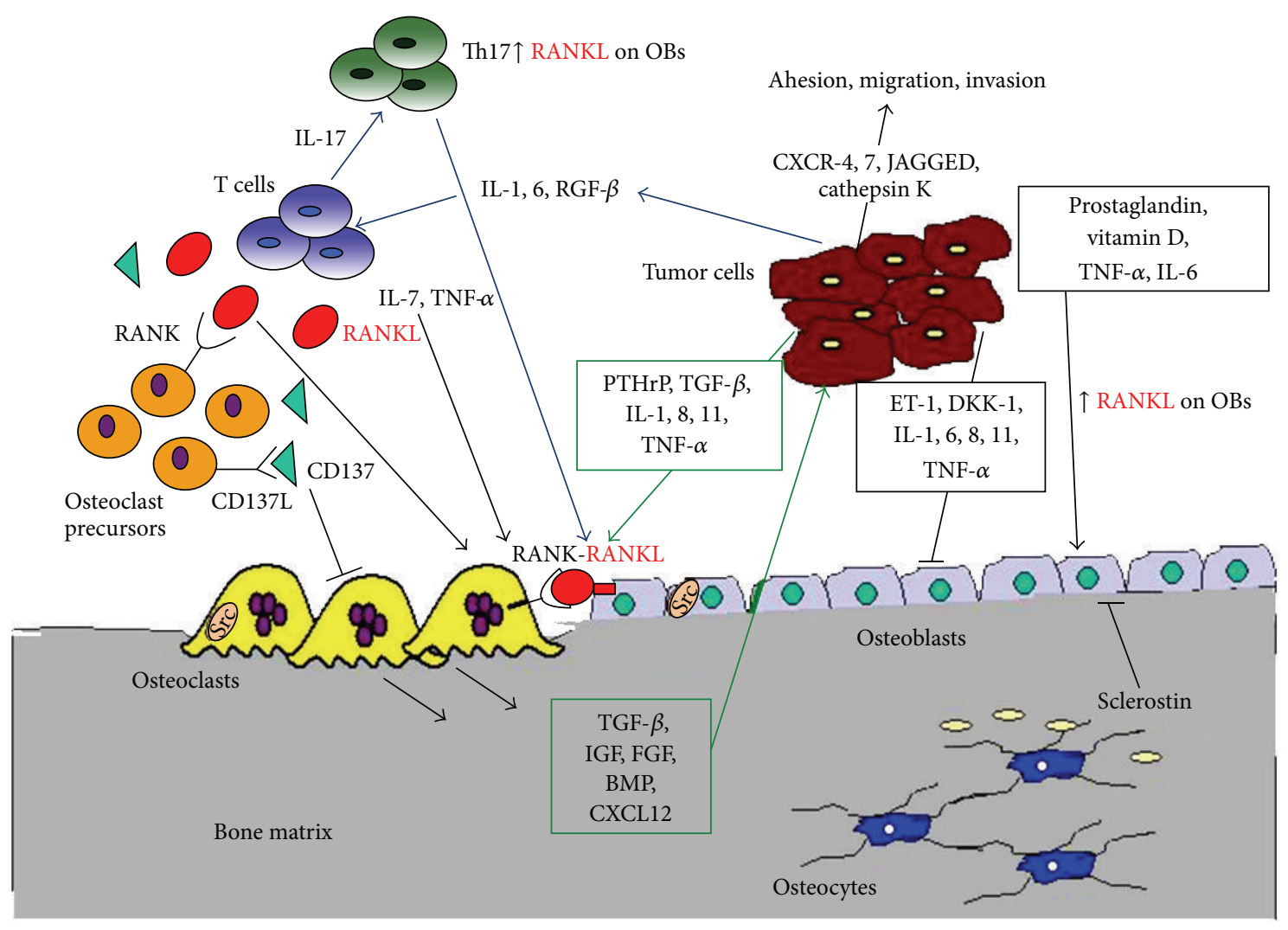

Figure 1: Molecular interactions among bone, tumor, and T cells.

develop further skeletal metastases within two years from the beginning of the treatment, suggesting that additional cells modulate bone tumor growth. These cells are T lymphocytes: tumor cell-derived IL-6, interleukin-1 (IL-1), and TGF- $\beta$ can drive T-cell differentiation towards a Thl7 secretory helpercell phenotype capable of inducing RANKL production by OBs and OC activation through interleukin-17 (IL-17) production (Figure 1) [42]. In breast cancer patients, memory $\mathrm{T}$ cells have been found in the bone marrow, suggesting their role in cancer immune surveillance [43]. Moreover, the RANKL-RANK interaction between helper $\mathrm{T}$ cells and breast cancer cells promotes invasion, dissemination, and metastasis formation from orthotopic syngeneic mouse mammary tumor virus-Erbb2 tumors in immunocompetent mice [44].

Noteworthy, some antibone metastatic therapies also showed immunomodulatory effects: the blockade of TGF$\beta$ at metastatic sites may locally activate an antitumor $\mathrm{T}$ cell response because normally TGF- $\beta$, released in bone marrow by OC activity, inhibits T-cell proliferation [45]. Zoledronic acid, used as antiresorptive agent, can activate cytotoxic $\gamma / \delta$-T cells and inhibit populations of myeloidderived cells with T-cell-suppressor capabilities [46]. Zhang and colleagues provide compelling evidence that a condition of immune deficiency can interfere with the antitumor effects of OC blockade [41]. Modulation of antitumor Tcell response alters tumor growth in bone, indeed Lyn(-/-) mice, which have more numerous OCs and a hyperactive myeloid population with an increased T-cell responses, had reduced tumor growth in bone despite enhanced osteolysis. Lyn is a member of the Src family tyrosine kinases and it is involved in the downregulation of different intracellular pathways, including the PLC $\gamma 2$ activation, which regulates the OC formation and function, thus Lyn inhibits OC differentiation [47]. PLC $\gamma 2(-/-)$ mice, which have dysfunctional OCs and impaired dendritic cell-mediated T-cell activation, had increased bone tumor burden despite protection from bone loss. Importantly, injection of antigen-specific wildtype cytotoxic $\mathrm{CD} 8(+) \mathrm{T}$ cells in both these mice models reduces the growth of tumor cells in the bone, regardless of OC functionality. According to these data, $T$ cells seem to be critical regulators of tumor growth in bone, in particular their activation diminishes bone metastases, whereas their depletion enhances them, even in the presence of zoledronic acid.

Further proves of the direct dialogue between $\mathrm{T}$ cells and OCs derive by studies conducted on the PBMCs of patients affected by solid tumors with bone metastases, which show an increase of circulating OC precursors compared with both healthy controls and cancer patients without bone metastases [48]. These OC precursors differentiate into mature, multinucleated, and bone resorbing OCs in vitro, without adding proosteoclastogenic factors. This osteoclastogenesis is dependent on T cells that release TNF- $\alpha$ and RANKL, which act synergistically in promoting the OC differentiation. 
On the other hand, T-cell depletion does not allow the differentiation of PBMCs into OCs without adding M-CSF and RANKL [48].

As previously reported, IL-7 is an important regulator of the interaction between bone and immune system. Many studies report a role of IL-7 in bone homeostasis in particular to bone loss in oestrogen deficiency conditions $[22,49,50]$. Other works support an active role of IL-7 in promoting bone lesions from solid tumors and multiple myeloma [24, 25, 51, 52]. In culture of PBMCs derived from patients with bone metastases, IL-7 is mainly released by B cells, and it directly sensitises $\mathrm{T}$ cells to produce proosteoclastogenic factors, such as TNF- $\alpha$ and RANKL, which enhance spontaneous osteoclastogenesis [25]. Moreover, in bone metastatic patients IL-7 sera levels were found significantly higher than in nonbone metastatic patients and in healthy controls [25, 52]. This serum IL-7 increase seems to directly depend on tumor production: a strong IL-7 expression was detected in tumor masses originated in a human-in-mice model of bone metastases and in human bone metastatic biopsies [53].

Prostate cancer is typically characterised by the presence of osteoblastic lesions with underlying osteolytic area [54]. The osteolytic activity is explained by an increase in serum RANKL/OPG ratio in prostate cancer patients, thus an enhanced OC formation plays an active role in bone forming lesions. The RANKL increment depends at least in part on the increased IL-7 production from T and B cells, but unlike other solid tumors, IL-7 expression is not significantly different in prostate cancer patients and in normal controls [51]. In the early phase of prostate cancer bone metastases, the increased OC activity also depends on Wnt agonist Dickkopf1 (DKK-1) that inhibits OBs, favouring lytic lesions [55]. In a subsequent phase, there is an increase in endothelin-1 (ET1), which stimulates OBs and inhibits OCs, by decreasing the transcription of DKK-1 [56]. ET-1 is also expressed by breast cancer cells, explaining the presence of mixed bone lesions in advanced disease.

\section{Molecular Targets for the Treatment of Bone Metastases}

In the last years, thanks to the identification of the above reported factors produced by bone, tumor, and immune system cells, many novel agents able to prevent or block bone metastases have been developed. The identification of the RANKL/RANK/OPG signalling pathway provided the basis for the development of new therapeutic molecules, such as denosumab, the first fully human antibody anti-RANKL. The first attempt to interfere with RANKL signalling to block and/or prevent bone metastases was the use of OPG or OPGFc $[57,58]$. Unlike OPG-Fc, denosumab specifically binds to RANKL, without interfering with other TNF ligands. In clinical trials, it resulted able to prevent the homing of RANK expressing tumor cells to bone and to inhibit the formation of bone lesions [59], thus it has been approved for the treatment of bone metastases in prostate and breast cancer patients. Even though denosumab inhibits bone resorption in patients refractory to bisphosphonate therapy [60] and ameliorates the quality of life of patients bearing bone metastases, it dose not improve the overall survival of these patients has not been demonstrated.

Src (sarcoma) is a protooncogene encoding a tyrosine kinase; it is highly expressed on OCs, where it is activated, during the bone resorption, in the process of RANK signalling and after the integrin binding. Src signalling is essential for the organization of OC cytoskeleton [61] and its lack does not allow to build an intact ruffled border causing osteopetrosis [62]. Src also inhibits RUNX2-regulated genes, thus it negatively regulates OBs [63]. Moreover, increased Src expression and activity associated with metastatic ability of many tumors [64]. Due to Src action on OCs, OBs and cancer cells, several Src inhibitors are currently being evaluated in clinical trials to test their ability in blocking skeletal lesions $[65,66]$.

Cathepsin $\mathrm{K}$ is expressed by mature OCs, being fundamental for their resorptive acitivity. It is expressed in different tumors $[67,68]$ and it promotes osteolytic lesions in a mouse model of prostate cancer, whereas its inhibition reduces the skeletal lesion in a breast cancer model [69]. Cathepsin $\mathrm{K}$ inhibitors have been investigated and tested for bone metastases treatment [70], but currently there are ongoing clinical trials only for its use in osteoporosis.

Activin A, a member of the TGF- $\beta$ family, has been described to stimulate osteoclastogenesis and to inhibit OBs. Increased levels of activin A have been described in the bone marrow plasma of breast and prostate cancer with bone metastases [71]. In mouse models of human breast cancer and multiple myeloma, activin A inhibition is effective to prevent bone lesions [72, 73].

DKK-1 is inhibitor of Wnt signalling pathway that regulates $\mathrm{OB}$ development and activity and indirectly osteoclastogenesis by shifting the RANKL-to-OPG ratio towards OPG [74]. The inhibition of DKK-1 could be an effective method to prevent osteolytic bone lesions, thus its inhibitor has been tested with encouraging results in experimental model of multiple myeloma and breast cancer $[75,76]$. An anti- DKK-1 antibody (BHQ-880) is currently under investigation in three clinical trials.

Inhibition of ET-1 signalling is a target for the inhibition of osteoblastic metastases in advanced prostate cancer, and both anti-ET-1 antibodies and blockade of ET-A receptor have been proposed $[77,78]$.

\section{Conclusion}

It has become increasingly evident that tumor cells interact with bone microenvironment and induce the activation of the immune system cells, which in turn release many factors able to promote bone metastases (Figure 1). This interplay creates cellular feedback loops with activation of multiple signalling, providing different potential molecular targets for the prevention and treatment of bone metastases.

\section{References}

[1] R. E. Coleman, "Clinical features of metastatic bone disease and risk of skeletal morbidity," Clinical Cancer Research, vol. 12, no. 20, part 2, pp. 6243s-6249s, 2006. 
[2] R. E. Coleman, "Metastatic bone disease: clinical features, pathophysiology and treatment strategies," Cancer Treatment Reviews, vol. 27, no. 3, pp. 165-176, 2001.

[3] G. D. Roodman, "Mechanisms of bone metastasis," The New England Journal of Medicine, vol. 350, no. 16, pp. 1655-1664, 2004.

[4] S. L. Teitelbaum, "Bone resorption by osteoclasts," Science, vol. 289, no. 5484, pp. 1504-1508, 2000.

[5] G. D. Roodman, "Cell biology of the osteoclast," Experimental Hematology, vol. 27, no. 8, pp. 1229-1241, 1999.

[6] C. J. Logothetis and S. H. Lin, "Osteoblasts in prostate cancer metastasis to bone," Nature Reviews Cancer, vol. 5, no. 1, pp. 2128, 2005.

[7] H. Takayanagi, "New developments in osteoimmunology," $\mathrm{Na}$ ture Reviews Rheumatology, vol. 8, no. 11, pp. 684-689, 2012.

[8] S. Kasagi and W. Chen, "TGF-betal on osteoimmunology and the bone component cells," Cell \& Bioscience, vol. 3, no. 1, article 4, 2013.

[9] P. J. Kostenuik and V. Shalhoub, "Osteoprotegerin: a physiological and pharmacological inhibitor of bone resorption," Current Pharmaceutical Design, vol. 7, no. 8, pp. 613-635, 2001.

[10] L. C. Hofbauer, C. A. Kühne, and V. Viereck, "The OPG/ RANKL/RANK system in metabolic bone diseases," Journal of Musculoskeletal Neuronal Interactions, vol. 4, no. 3, pp. 268-275, 2004.

[11] D. Santini, G. Perrone, I. Roato et al., "Expression pattern of receptor activator of NFkappaB (RANK) in a series of primary solid tumors and related bone metastases," Journal of Cellular Physiology, vol. 226, no. 3, pp. 780-784, 2011.

[12] L. C. Hofbauer, S. Khosla, C. R. Dunstan, D. L. Lacey, W. J. Boyle, and B. L. Riggs, "The roles of osteoprotegerin and osteoprotegerin ligand in the paracrine regulation of bone resorption," Journal of Bone and Mineral Research, vol. 15, no. 1, pp. 2-12, 2000.

[13] R. Pacifici, "The immune system and bone," Archives of Biochemistry and Biophysics, vol. 503, no. 1, pp. 41-53, 2010.

[14] H. Takayanagi, K. Ogasawara, S. Hida et al., "T-cell-mediated regulation of osteoclastogenesis by signalling cross-talk between RANKL and IFN- $\gamma$," Nature, vol. 408, no. 6812, pp. 600-605, 2000.

[15] K. Sato, T. Satoh, K. Shizume et al., "Prolonged decrease of serum calcium concentration by murine $\gamma$-interferon in hypercalcemic, human tumor (EC-GI)-bearing nude mice," Cancer Research, vol. 52, no. 2, pp. 444-449, 1992.

[16] Y. Gao, F. Grassi, M. R. Ryan et al., "IFN- $\gamma$ stimulates osteoclast formation and bone loss in vivo via antigen-driven $\mathrm{T}$ cell activation," Journal of Clinical Investigation, vol. 117, no. 1, pp. 122-132, 2007.

[17] S. Cenci, G. Toraldo, M. N. Weitzmann et al., "Estrogen deficiency induces bone loss by increasing $\mathrm{T}$ cell proliferation and lifespan through IFN- $\gamma$-induced class II transactivator," Proceedings of the National Academy of Sciences of the United States of America, vol. 100, no. 18, pp. 10405-10410, 2003.

[18] S. Abbas and Y. Abu-Amer, "Dominant-negative $\mathrm{I} \kappa \mathrm{B}$ facilitates apoptosis of osteoclasts by tumor necrosis factor- $\alpha$," Journal of Biological Chemistry, vol. 278, no. 22, pp. 20077-20082, 2003.

[19] R. Pacifici, "Estrogen deficiency, T cells and bone loss," Cellular Immunology, vol. 252, no. 1-2, pp. 68-80, 2008.

[20] A. E. Namen, S. Lupton, K. Hjerrild et al., "Stimulation of B-cell progenitors by cloned murine interleukin-7," Nature, vol. 333, no. 6173 , pp. 571-573, 1988.
[21] C. Miyaura, Y. Onoe, M. Inada et al., "Increased B-lymphopoiesis by interleukin 7 induces bone loss in mice with intact ovarian function: similarity to estrogen deficiency," Proceedings of the National Academy of Sciences of the United States of America, vol. 94, no. 17, pp. 9360-9365, 1997.

[22] M. N. Weitzmann, C. Roggia, G. Toraldo, L. Weitzmann, and R. Pacifici, "Increased production of IL-7 uncouples bone formation from bone resorption during estrogen deficiency," Journal of Clinical Investigation, vol. 110, no. 11, pp. 1643-1650, 2002.

[23] G. Toraldo, C. Roggia, W. P. Qian, R. Pacific, and M. N. Weitzmann, "IL-7 induces bone loss in vivo by induction of receptor activator of nuclear factor $\kappa \mathrm{B}$ ligand and tumor necrosis factor $\alpha$ from T cells," Proceedings of the National Academy of Sciences of the United States of America, vol. 100, no. 1, pp. 125-130, 2003.

[24] N. Giuliani, S. Colla, R. Sala et al., "Human myeloma cells stimulate the receptor activator of nuclear factor- $\kappa \mathrm{B}$ ligand (RANKL) in T lymphocytes: a potential role in multiple myeloma bone disease," Blood, vol. 100, no. 13, pp. 4615-4621, 2002.

[25] I. Roato, G. Brunetti, E. Gorassini et al., "IL-7 up-regulates TNF$\alpha$-dependent osteoclastogenesis in patients affected by solid tumor," PLoS One, vol. 1, no. 1, article e124, 2006.

[26] P. D’Amelio, A. Grimaldi, S. Di Bella et al., "Estrogen deficiency increases osteoclastogenesis up-regulating T cells activity: a key mechanism in osteoporosis," Bone, vol. 43, no. 1, pp. 92-100, 2008.

[27] J. R. Kiesel, Z. S. Buchwald, and R. Aurora, "Cross-presentation by osteoclasts induces FoxP3 in $\mathrm{CD}^{+}$T cells," Journal of Immunology, vol. 182, no. 9, pp. 5477-5487, 2009.

[28] R. Senthilkumar and H. W. Lee, "CD137L- and RANKLmediated reverse signals inhibit osteoclastogenesis and T lymphocyte proliferation," Immunobiology, vol. 214, no. 2, pp. 153161, 2009.

[29] S. Paget, "The distribution of secondary growths in cancer of the breast,” The Lancet, vol. 133, no. 3421, pp. 571-573, 1889.

[30] R. Salcedo and J. J. Oppenheim, "Role of chemokines in angiogenesis: CXCL12/SDF-1 and CXCR4 interaction, a key regulator of endothelial cell responses," Microcirculation, vol.10, no. 3-4, pp. 359-370, 2003.

[31] J. Wang, Y. Shiozawa, J. Wang et al., "The role of CXCR7/RDC1 as a chemokine receptor for CXCL12/SDF-1 in prostate cancer," Journal of Biological Chemistry, vol. 283, no. 7, pp. 4283-4294, 2008.

[32] P. V. Hauschka, A. E. Mavrakos, M. D. Iafrati, S. E. Doleman, and M. Klagsbrun, "Growth factors in bone matrix. Isolation of multiple types by affinity chromatography on heparinsepharose," Journal of Biological Chemistry, vol. 261, no. 27, pp. 12665-12674, 1986.

[33] N. Sethi, X. Dai, C. G. Winter, and Y. Kang, "Tumor-derived JAGGED1 promotes osteolytic bone metastasis of breast cancer by engaging notch signaling in bone cells," Cancer Cell, vol. 19, no. 2, pp. 192-205, 2011.

[34] S. Santagata, F. Demichelis, A. Riva et al., "JAGGED1 expression is associated with prostate cancer metastasis and recurrence," Cancer Research, vol. 64, no. 19, pp. 6854-6857, 2004.

[35] S. Chiba, "Notch signaling in stem cell systems," Stem Cells, vol. 24, no. 11, pp. 2437-2447, 2006.

[36] A. Bellahcene, M. P. Merville, and V. Castronovo, "Expression of bone sialoprotein, a bone matrix protein, in human breast cancer," Cancer Research, vol. 54, no. 11, pp. 2823-2826, 1994. 
[37] A. Bellahcene and V. Castronovo, "Increased expression of osteonectin and osteopontin, two bone matrix proteins, in human breast cancer," American Journal of Pathology, vol. 146, no. 1, pp. 95-100, 1995.

[38] K. S. Koeneman, F. Yeung, and L. W. Chung, "Osteomimetic properties of prostate cancer cells: a hypothesis supporting the predilection of prostate cancer metastasis and growth in the bone environment," Prostate, vol. 39, no. 4, pp. 246-261, 1999.

[39] D. L. Lin, C. P. Tarnowski, J. Zhang et al., "Bone metastatic LNCaP-derivative C4-2B prostate cancer cell line mineralizes in vitro," Prostate, vol. 47, no. 3, pp. 212-221, 2001.

[40] R. F. Cox, A. Jenkinson, K. Pohl et al., "Osteomimicry of mammary adenocarcinoma cells in vitro, increased expression of bone matrix proteins and proliferation within a 3D collagen environment," PLoS One, vol. 7, no. 7, Article ID e41679, 2012.

[41] K. Zhang, S. Kim, V. Cremasco et al., " $\mathrm{CD} 8^{+} \mathrm{T}$ cells regulate bone tumor burden independent of osteoclast resorption," Cancer Research, vol. 71, no. 14, pp. 4799-4808, 2011.

[42] P. Miossec, T. Korn, and V. K. Kuchroo, "Interleukin-17 and type 17 helper T cells," The New England Journal of Medicine, vol. 361, no. 9, pp. 848-898, 2009.

[43] M. Feuerer, M. Rocha, L. Bai et al., "Enrichment of memory T cells and other profound immunological changes in the bone marrow from untreated breast cancer patients," International Journal of Cancer, vol. 92, no. 1, pp. 96-105, 2001.

[44] W. Tan, W. Zhang, A. Strasner et al., "Tumour-infiltrating regulatory $\mathrm{T}$ cells stimulate mammary cancermetastasis through RANKL-RANK signalling," Nature, vol. 470, no. 7335, pp. 548553, 2011.

[45] S. H. Wrzesinski, Y. Y. Wan, and R. A. Flavell, "Transforming growth factor- $\beta$ and the immune response: implications for anticancer therapy," Clinical Cancer Research, vol. 13, no. 18, part 1, pp. 5262-5270, 2007.

[46] K. Schilbach, A. Geiselhart, and R. Handgretinger, "Induction of proliferation and augmented cytotoxicity of $\gamma \delta \mathrm{T}$ lymphocytes by bisphosphonate clodronate," Blood, vol. 97, no. 9, pp. 2917-2918, 2001.

[47] S. H. Yoon, Y. Lee, H. J. Kim et al., "Lyn inhibits osteoclast differentiation by interfering with PLC $\gamma 1$-mediated $\mathrm{Ca}^{2+}$ signaling," FEBS Letters, vol. 583, no. 7, pp. 1164-1170, 2009.

[48] I. Roato, M. Grano, G. Brunetti et al., "Mechanisms of spontaneous osteoclastogenesis in cancer with bone involvement," FASEB Journal, vol. 19, no. 2, pp. 228-230, 2005.

[49] S. Cenci, M. N. Weitzmann, C. Roggia et al., "Estrogen deficiency induces bone loss by enhancing T-cell production of TNF- $\alpha$," Journal of Clinical Investigation, vol. 106, no. 10, pp. 1229-1237, 2000.

[50] M. R. Ryan, R. Shepherd, J. K. Leavey et al., "An IL-7-dependent rebound in thymic $\mathrm{T}$ cell output contributes to the bone loss induced by estrogen deficiency," Proceedings of the National Academy of Sciences of the United States of America, vol. 102, no. 46, pp. 16735-16740, 2005.

[51] I. Roato, P. D’Amelio, E. Gorassini et al., "Osteoclasts are active in bone forming metastases of prostate cancer patients," PLoS One, vol. 3, no. 11, Article ID e3627, 2008.

[52] I. Roato, E. Gorassini, L. Buffoni et al., "Spontaneous osteoclastogenesis is a predictive factor for bone metastases from nonsmall cell lung cancer," Lung Cancer, vol. 61, no. 1, pp. 109-116, 2008.

[53] I. Roato, D. Caldo, L. Godio et al., "Bone invading NSCLC cells produce IL-7: mice model and human histologic data," $B M C$ Cancer, vol. 10, article 12, 2010.
[54] E. T. Keller, J. Zhang, C. R. Cooper et al., "Prostate carcinoma skeletal metastases: cross-talk between tumor and bone," Cancer and Metastasis Reviews, vol. 20, no. 3-4, pp. 333-349, 2001.

[55] C. L. Hall, S. Kang, O. A. MacDougald, and E. T. Keller, "Role of Wnts in prostate cancer bone metastases," Journal of Cellular Biochemistry, vol. 97, no. 4, pp. 661-672, 2006.

[56] G. A. Clines, K. S. Mohammad, Y. Bao et al., "Dickkopf homolog 1 mediates endothelin-1-stimulated new bone formation," Molecular Endocrinology, vol. 21, no. 2, pp. 486-498, 2007.

[57] J. Zhang, J. Dai, Y. Qi et al., "Osteoprotegerin inhibits prostate cancer-induced osteoclastogenesis and prevents prostate tumor growth in the bone," Journal of Clinical Investigation, vol. 107, no. 10, pp. 1235-1244, 2001.

[58] S. Morony, C. Capparelli, I. Sarosi, D. L. Lacey, C. R. Dunstan, and P. J. Kostenuik, "Osteoprotegerin inhibits osteolysis and decreases skeletal tumor burden in syngeneic and nude mouse models of experimental bone metastasis," Cancer Research, vol. 61, no. 11, pp. 4432-4436, 2001.

[59] A. Lipton, G. G. Steger, J. Figueroa et al., "Randomized activecontrolled phase II study of denosumab efficacy and safety in patients with breast cancer-related bone metastases," Journal of Clinical Oncology, vol. 25, no. 28, pp. 4431-4437, 2007.

[60] J. J. Body, T. Facon, R. E. Coleman et al., "A study of the biological receptor activator of nuclear factor- $\kappa$ ligand inhibitor, denosumab, in patients with multiple myeloma or bone metastases from breast cancer," Clinical Cancer Research, vol. 12, no. 4, pp. 1221-1228, 2006.

[61] T. Miyazaki, A. Sanjay, L. Neff, S. Tanaka, W. C. Horne, and R. Baron, "Src kinase activity is essential for osteoclast function," Journal of Biological Chemistry, vol. 279, no. 17, pp. 17660-17666, 2004.

[62] P. Soriano, C. Montgomery, R. Geske, and A. Bradley, “Targeted disruption of the c-src proto-oncogene leads to osteopetrosis in mice," Cell, vol. 64, no. 4, pp. 693-702, 1991.

[63] S. K. Zaidi, A. J. Sullivan, R. Medina et al., "Tyrosine phosphorylation controls Runx2-mediated subnuclear targeting of YAP to repress transcription," EMBO Journal, vol. 23, no. 4, pp. 790799, 2004.

[64] M. C. Frame, "Src in cancer: deregulation and consequences for cell behaviour," Biochimica et Biophysica Acta, vol. 1602, no. 2, pp. 114-130, 2002.

[65] R. A. Hannon, G. Clack, M. Rimmer et al., "Effects of the Src kinase inhibitor saracatinib (azd0530) on bone turnover in healthy men: a randomized, double-blind, placebo-controlled, multiple-ascending-dose phase I trial," Journal of Bone and Mineral Research, vol. 25, no. 3, pp. 463-471, 2010.

[66] A. Gucalp, J. A. Sparano, J. Caravelli et al., "Phase II trial of saracatinib (AZD0530), an oral SRC-inhibitor for the treatment of patients with hormone receptor-negative metastatic breast cancer," Clinical Breast Cancer, vol. 11, no. 5, pp. 306-311, 2011.

[67] K. D. Brubaker, R. L. Vessella, L. D. True, R. Thomas, and E. Corey, "Cathepsin K mRNA and protein expression in prostate cancer progression," Journal of Bone and Mineral Research, vol. 18, no. 2, pp. 222-230, 2003.

[68] A. J. Littlewood-Evans, G. Bilbe, W. B. Bowler et al., "The osteoclast-associated protease cathepsin $\mathrm{K}$ is expressed in human breast carcinoma," Cancer Research, vol. 57, no. 23, pp. 5386-5390, 1997.

[69] C. Le Gall, A. Bellahcène, E. Bonnelye et al., "A cathepsin K inhibitor reduces breast cancer-induced osteolysis and skeletal tumor burden," Cancer Research, vol. 67, no. 20, pp. 9894-9902, 2007. 
[70] A. B. Jensen, C. Wynne, G. Ramirez et al., "The cathepsin $\mathrm{K}$ inhibitor odanacatib suppresses bone resorption in women with breast cancer and established bone metastases: results of a 4-week, double-blind, randomized, controlled trial," Clinical Breast Cancer, vol. 10, no. 6, pp. 452-458, 2010.

[71] G. Leto, L. Incorvaia, G. Badalamenti et al., "Activin A circulating levels in patients with bone metastasis from breast or prostate cancer," Clinical and Experimental Metastasis, vol. 23, no. 2, pp. 117-122, 2006.

[72] A. D. Chantry, D. Heath, A. W. Mulivor et al., "Inhibiting activin-A signaling stimulates bone formation and prevents cancer-induced bone destruction in vivo," Journal of Bone and Mineral Research, vol. 25, no. 12, pp. 2357-2370, 2010.

[73] S. Vallet, S. Mukherjee, N. Vaghela et al., "Activin A promotes multiple myeloma-induced osteolysis and is a promising target for myeloma bone disease," Proceedings of the National Academy of Sciences of the United States of America, vol. 107, no. 11, pp. 5124-5129, 2010.

[74] G. J. Spencer, J. C. Utting, S. L. Etheridge, T. R. Arnett, and P. G. Genever, "Wnt signalling in osteoblasts regulates expression of the receptor activator of $\mathrm{NF} \kappa \mathrm{B}$ ligand and inhibits osteoclastogenesis in vitro," Journal of Cell Science, vol. 119, no. 7, pp. 12831296, 2006.

[75] D. J. Heath, A. D. Chantry, C. H. Buckle et al., "Inhibiting dickkopf-1 (Dkkl) removes suppression of bone formation and prevents the development of osteolytic bone disease in multiple myeloma," Journal of Bone and Mineral Research, vol. 24, no. 3, pp. 425-436, 2009.

[76] L. Qiao, Z. L. Xu, T. J. Zhao, L. H. Ye, and X. D. Zhang, "Dkk1 secreted by mesenchymal stem cells inhibits growth of breast cancer cells via depression of Wnt signalling," Cancer Letters, vol. 269, no. 1, pp. 67-77, 2008.

[77] J. W. Chiao, B. S. Moonga, Y. M. Yang et al., "Endothelin-1 from prostate cancer cells is enhanced by bone contact which blocks osteoclastic bone resorption," British Journal of Cancer, vol. 83, no. 3, pp. 360-365, 2000.

[78] J. J. Yin, K. S. Mohammad, S. M. Kakonen et al., "A causal role for endothelin-1 in the pathogenesis of osteoblastic bone metastases," Proceedings of the National Academy of Sciences of the United States of America, vol. 100, no. 19, pp. 10954-10959, 2003. 


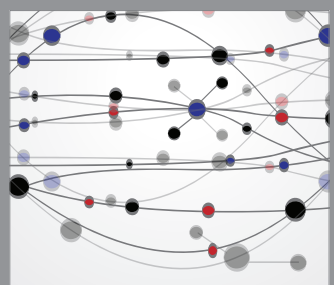

The Scientific World Journal
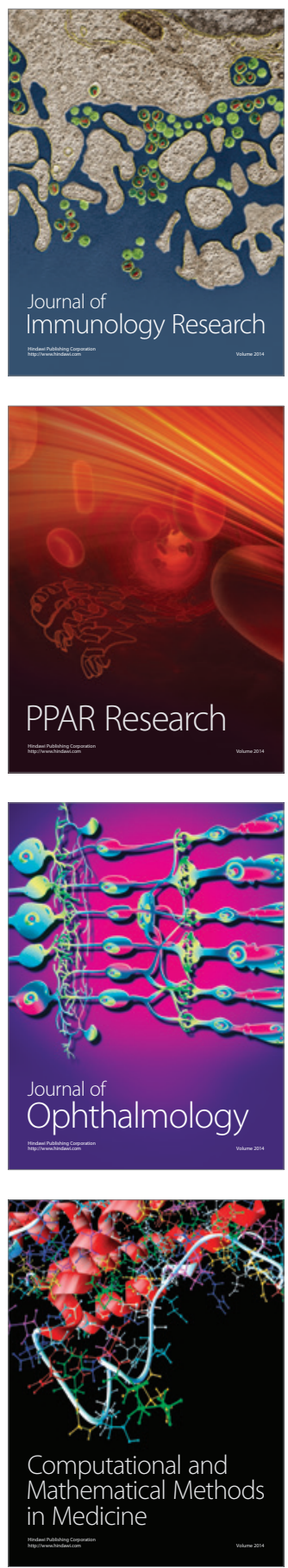

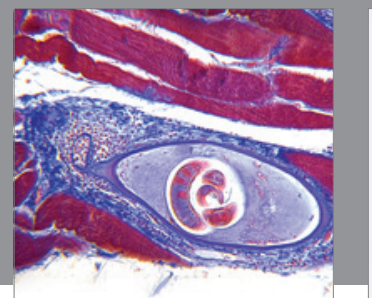

Gastroenterology

Research and Practice
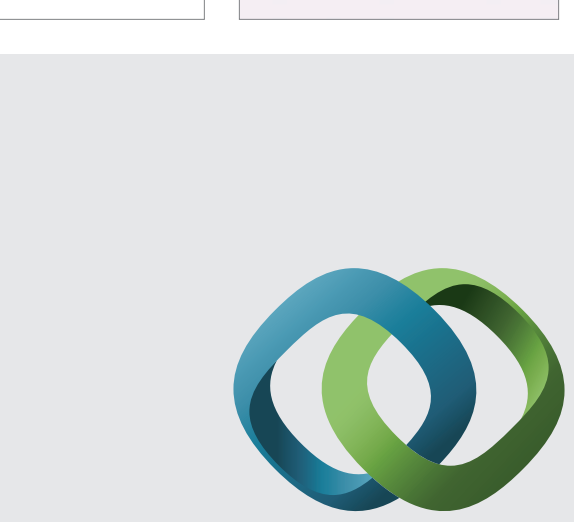

\section{Hindawi}

Submit your manuscripts at

http://www.hindawi.com
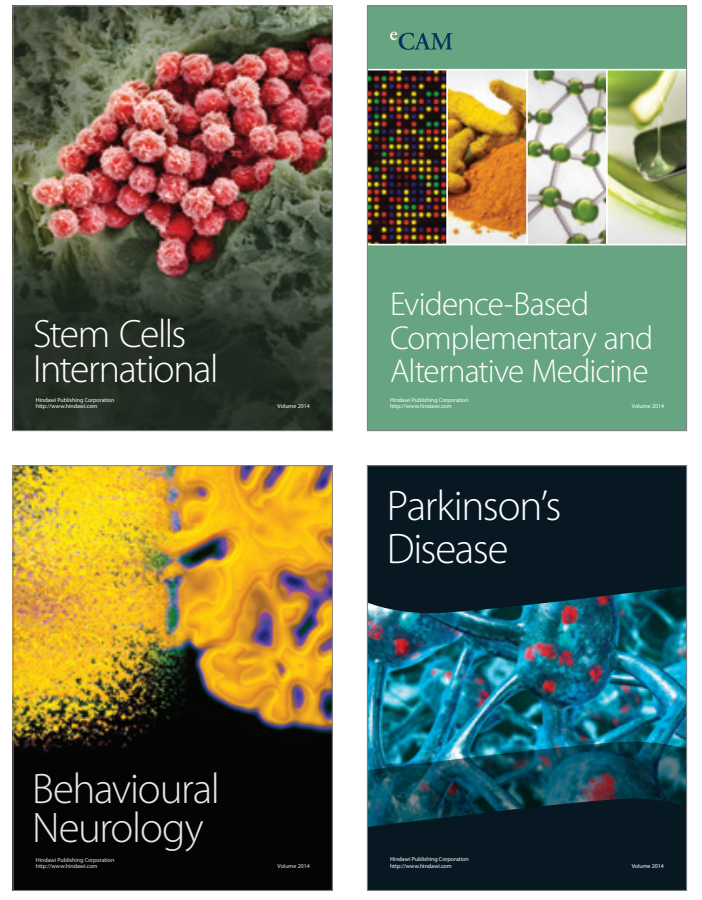
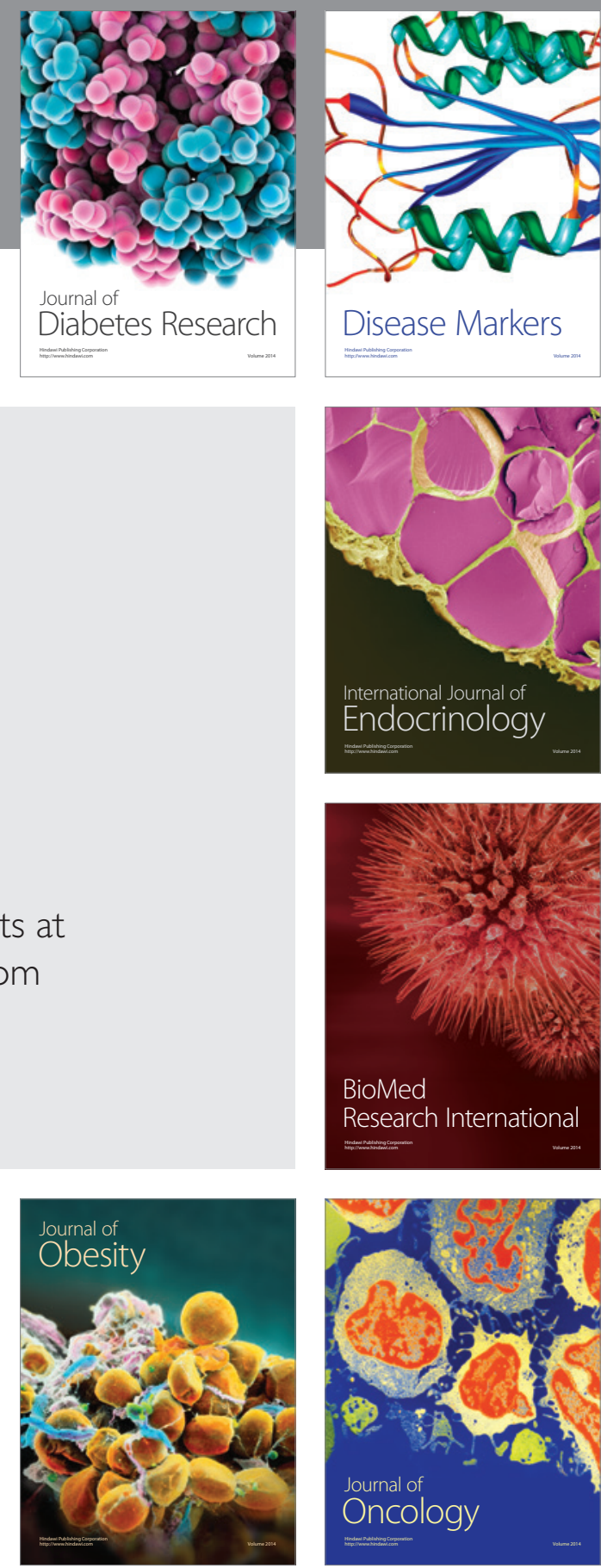

Disease Markers
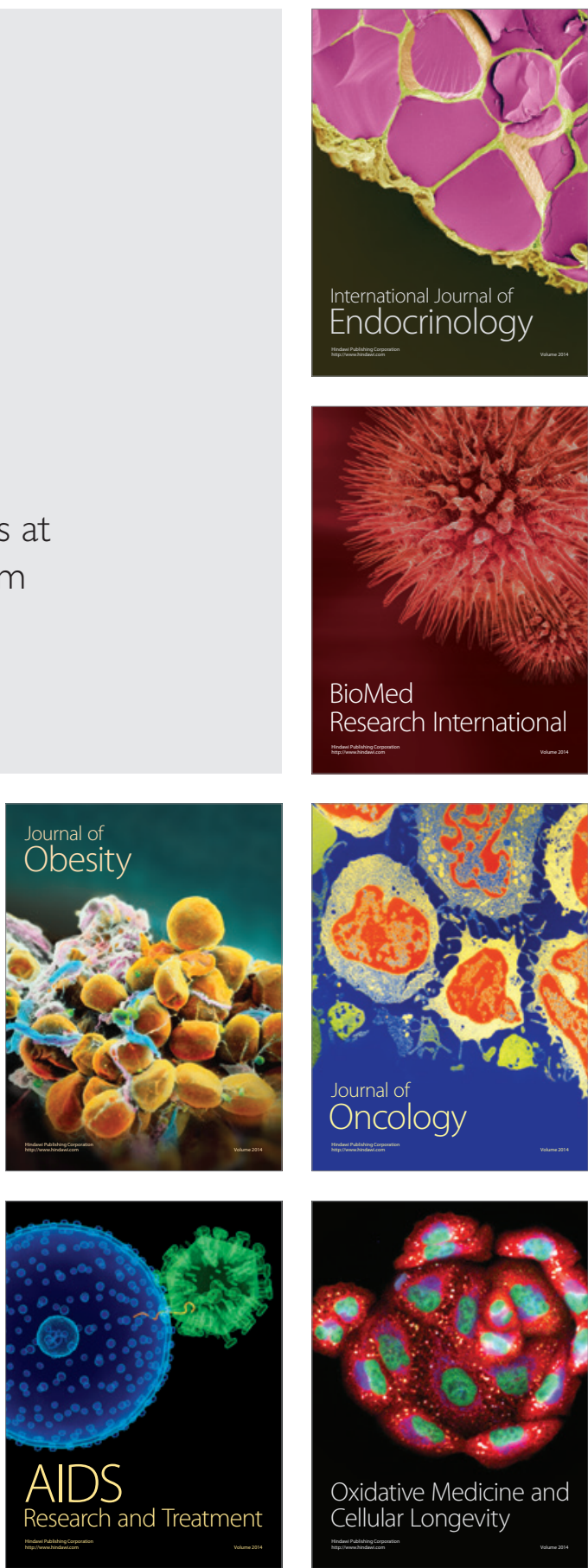